\title{
Primary Lumbo-sacral Spinal Epidural Non-Hodgkin's Lymphoma: A Case Report and Review of Literature
}

\author{
Rahul Mally, Mayur Sharma, Shadma Khan, Vernon Velho \\ Department of Neurosurgery, Sir J. J. Group of Hospitals, Grant Medical College, Mumbai, Maharashtra, India
}

We present a case of 24-year-old male presented with low back pain radiating to the left lower limb, tingling numbness and weakness of 6 months duration. Magnetic resonance imaging scan with contrast reveals an extradural mass at lumbosacral region. Patient was operated with laminectomy and complete excision of the lesion was done. Patient's radicular pain relieved following the surgery and weakness also improved. Histopathology was suggestive of non-Hodgkin's lymphoma. Patient received chemotherapy which was followed by radiotherapy. Primary Non-Hodgkin's lymphoma of the lumbosacral spinal epidural tissue is an uncommon lesion. Lymphoma involves the central nervous system in $5-11 \%$ of cases either at presentation of the disease or during its course. The spinal epidural tissue is involved primarily in 0.1-3.3\% of cases with spinal cord compression being the commonest presentation. Excision of the lesion followed by chemotherapy and radiotherapy is required to achieve cure.

Key Words: Lumbosacral region, Spinal, Epidural, Non-Hodgkin's lymphoma

\section{Introduction}

We present a rare case of lumbo-sacral spinal epidural non-Hodgkin's lymphoma in a 24-year-old male, which on initial clinical and radiological examination was suggestive of a peripheral nerve sheath tumor. The lumbo-sacral spinal epidural space is an uncommon site for primary nonHodgkin's lymphomas, presenting with features of cauda equina compression [1-3]. These lesions are most common in middle aged males [1-3]. Mid thoracic spine is the most common site of involvement followed by lumbar and then by cervical spine [1-3].

\section{Case Report}

A 24-year-old, immunocompetent male was admitted with 6 months history of pain in left lower limb, tingling sensation for 2 months and weakness for 10 days. Pain was aching in nature and radiating from the buttocks to the knees. Pain aggravated on lying in left lateral position, walking, bending forward and was relieved in right lateral position.

On neurological examination, higher mental functions and cranial nerves were normal. Power was $5 / 5$ at left hip, $4 / 5$ at knee and ankle, and extensor/flexor hallucis longus were weak on left side. Power at rest of the joints was $5 / 5$. Sensory examination did not reveal any abnormality. Both superficial and deep tendon reflexes were normal. Gait was antalgic with weight bearing on right lower limb. Straight leg raising test was free on right side and restricted to $60^{\circ}$ on left side.

X-ray lumbo-sacral spine was normal. Magnetic resonance imaging (MRI) scan (Figs. 1-3) with contrast was

Received Jun 11, 2010; 1st Revised Jul 13, 2010; 2nd Revised Aug 3, 2010; Accepted Aug 5, 2010

Corresponding author: Mayur Sharma, MS

Department of Neurosurgery, Sir J. J. Group of Hospitals, Grant Medical College,

Byculla, Mumbai 400008, Maharashtra, India

Tel: +91-22-23735555 Ext: 2264, Fax: +91-22-23735599, E-mail: drmayur_sharma@yahoo.co.in 


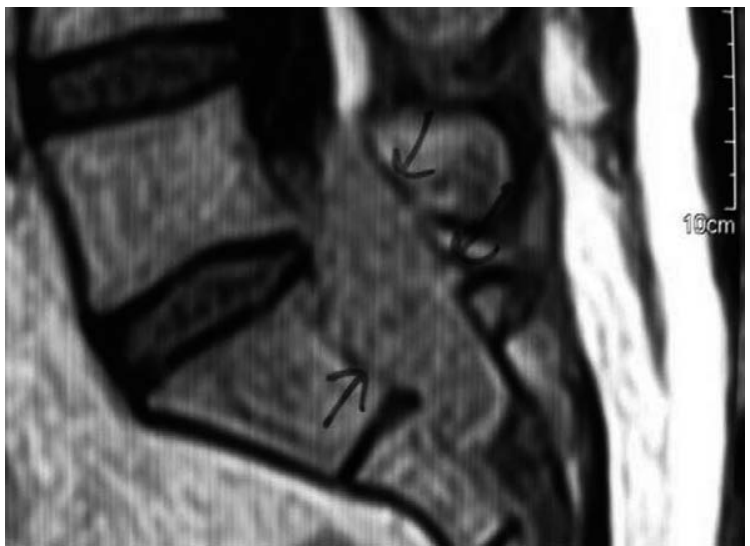

Fig. 1. Magnetic resonance imaging scan (sagittal view) showing well defined lobulated mass lesion L5-S1 to mid $\mathrm{S} 2$ level. The lesion was iso- to hypo-intense on T1 weighted images.

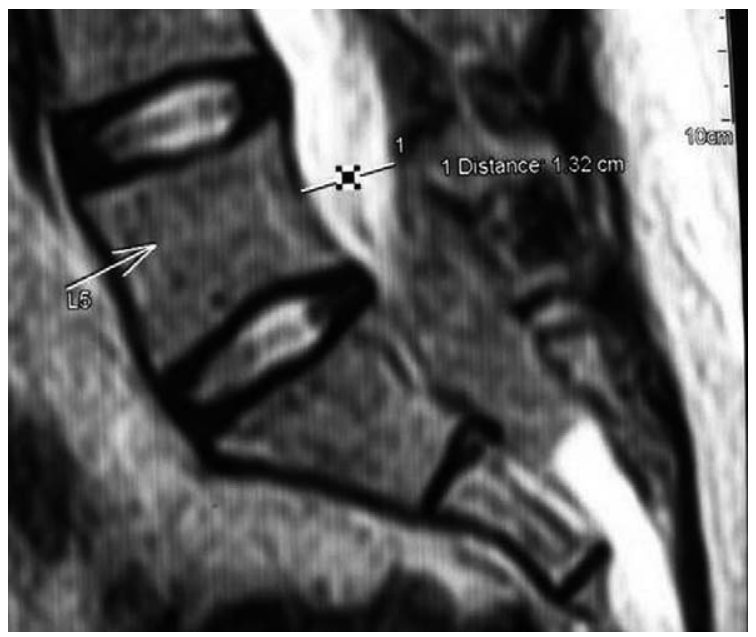

Fig. 2. Magnetic resonance imaging scan (sagittal view) showing well defined lobulated lesion. The lesion was isoto hypo-intense on $\mathrm{T} 2$ weighted images.

showing well defined lobulated extradural mass lesion of size $3.2 \times 3.0 \times 3.0 \mathrm{~cm}$ at L5-S1 to mid S2 level. The lesion was iso- to hypo-intense on $\mathrm{T} 1$ and $\mathrm{T} 2$ weighted images, hyper-intense on short T-1 inversion recovery and showed homogenous enhancement on post-contrast images.

Electrophysiological studies were suggestive of left L5 and bilateral S1 chronic radiculopathy.

Provisional pre-operative diagnosis was neurofibroma.

Patient was operated with L5-S2 laminectomy and total excision of the lesion. Intra-operatively, the lesion was extradural, fleshy, brownish and vascular. The lesion was densely adherent to the dura. Complete excision was achieved. Lesion was reported as chronic inflammatory tis-

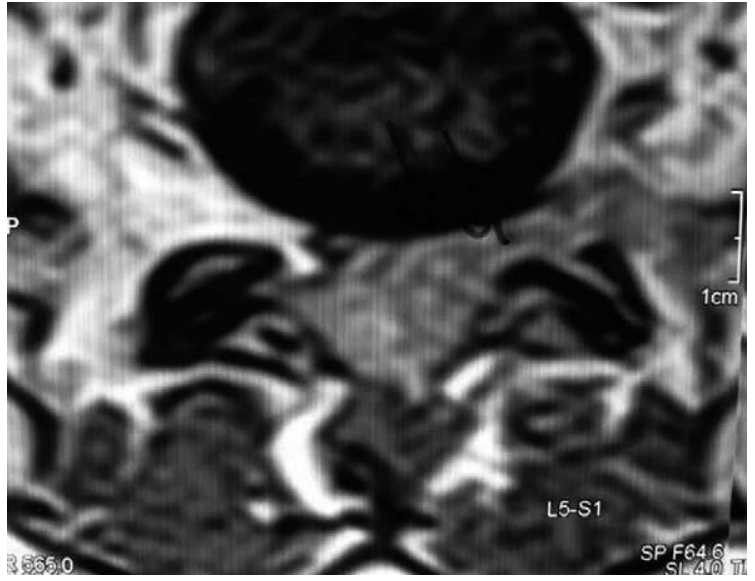

Fig. 3. Magnetic resonance imaging scan (axial view) showing well defined lesion in the intraspinal canal traversing the left neural foramina and compressing the left nerve root.

sue on frozen sections.

Histopathological examination showed atypical lymphoid cell proliferation comprising of large oval to irregular shaped tumor cells admixed with small mature looking reacting lymphoid cell population. On immunohistochemistry the atypical large tumor cells were positive for leukocyte common antigen (LCA) and CD20; while negative for CD138, CD30, and CD3.

Final diagnosis was high grade non-Hodgkin's lymphoma, diffuse large B cell lymphoma (DLBCL) immunophenotype.

Patient's radicular pain and tingling numbness had improved immediately following the surgery. Weakness had also improved gradually with physiotherapy to $4 / 5$ at all joints in left lower limb at the time of discharge.

Subsequently patient was worked up for systemic disease with MRI brain, computed tomography (CT) abdomen and pelvis, CT thorax, which were normal. Bone marrow and cerebrospinal fluid examinations were normal.

Beta-2 micro globulin was $1.74 \mathrm{mg} / \mathrm{l}$ (reference range, 0.83 to $1.15 \mathrm{mg} / \mathrm{l}$ ). Patient was staged as - IA-E and started on 6 cycles of cyclophophamide, adriamycin, vincristine and prednisolone (CHOP regimen) +10 intrathecal methoteraxate followed by involved field radiation therapy to cauda equina from L3/L4 junction to S3-45Gys/25\#s.

At 3 month follow up, patient was relieved of his radicular pain and tingling numbness. Weakness had also improved to $+4 / 5$ all joints in left lower limb. 


\section{Discussion}

Non-Hodgkin's lymphoma is an uncommon lesion involving the spinal epidural space. Only $9 \%$ of spinal epidural tumors are lymphomas. Thoracic spine (69\%) being the most common site followed by lumbar (27\%) and cervical spine $(4 \%)[1,2]$.

Only in $0.1-3.3 \%$ of lymphomas, spinal epidural tissue is involved primarily $[1,3]$. Hodgkin's lymphoma constitutes only $0.2 \%$ of these epidural lesions [1].

In contrast to 24-year-old male of our case, the usual age for presentation is fourth to fifth decade of life. There is male preponderance (66-70\%) with male : female ratio of $1.6: 1$ [1-3]. which corresponds to our case. In our case, patient was a young male without any systemic manifestations of the disease.

Controversy exists regarding the origin of primary epidural non-Hodgkin's lymphomas (PENHL). Rubinstein [4] demonstrated that epidural tissue contains lymphoid cells and proposed the theory of antigenic stimulation with transformation cascade. Some authors suggest that these tumors originate from paraspinal, vertebral, retroperitoneal tissues and enter the epidural space through the intervertebral foramina [1-4].

The most common triad of presentation includes lower limb weakness, localized back pain and bladder dysfunction [1-3]. In our case, the presenting feature was left lower limb weakness, pain and tingling numbness.

MRI scan reveals iso/hypointense lesion on both T1/T2 weighted images and homogenous enhancement on postcontrast images, extending over multiple vertebrae [5]. In our case also lesion had similar MRI features.

On histopathological examination, these tumors show atypical lymphoid cell proliferation. On immunohistochemistry, these tumor cells are positive for LCA and CD20; while negative for CD138, CD30, and CD3. DLBCL is the most common type at this site [6], which corresponds to our case. PENHLs are almost invariably of the B-cell type, although indolent B-cell and T-cell variants are rarely seen. DLBCL involving spinal epidural space constitutes $1.8 \%$ of all DLBCLs [7].

Patients presenting with features of spinal cord compression require surgical intervention for tissue diagnosis and decompression. These tumors rarely cause bony erosion. Aabo and Walbom-Jørgensen [8] have reported no difference in outcome between patients undergoing decompres- sive laminectomy and radiotherapy vs. those receiving spinal radiation only. Patients of PENHL who presented with paraplegia of recent origin and underwent decompressive laminectomy showed significant neurological improvement [2]. In our case, we have done L5-S2 decompressive laminectomy with total excision of lesion with significant neurological improvement.

As lymphomas are highly radiosensitive and chemo-sensitive tumors therefore chemo-radiotherapy remains the main stay of treatment. CHOP regime remains the gold standard of treatment [2]. Non-Hodgkin's lymphoma requires a dose of 3,500 cGy to 4,000 cGy delivered in 2025 fractions over a period of 3-4 weeks to achieve radical cure [9].

Our patient also received the similar treatment regimen.

Primary spinal extradural non-Hodgkin's lymphoma has poor prognosis for patients $>50$ years of age, with aggressive histological types and with paraplegia and bladder and bowel involvement [2]. Long term survival is favorable in young patients with surgical decompression followed by chemotherapy and radiotherapy. The overall mean survival of patients is $8-9$ months with less than $10 \%$ surviving one year [10].

Primary spinal epidural non-Hodgkin's lymphoma should be considered in the differential diagnosis of the patients who present with spinal cord or cauda equina compression with prodrome of back pain, followed by a rapid neurological deterioration. The plain spine radiographs are normal and MRI scan reveals an extradural lesion compressing the cord in such cases. Surgical excision of the lesion for the diagnosis followed by chemotherapy and radiotherapy should result in significant neurological improvement.

\section{Acknowledgements}

We would like to thank our Dean, Sir J. J. group of hospitals for allowing us to publish the hospital record.

\section{REFERENCES}

1. Mclain RF, Markman M. Cancer in the spine: comprehensive care. Totowa: Human Press, 2006. p. 111.

2. Kapoor R, Kumar V, Sharma SC. Primary extradural nonHodgkin's lymphoma. JK Science 2006;8:45-8.

3. Lim CC, Chong BK. Spinal epidural non-Hodgkin's lymphoma: case reports of three patients presenting with spinal cord compression. Singapore Med J 1996;37:497-500. 
4. Rubinstein LJ. Tumor of the nervous system. Arch of tumor pathology. Facide 6. Tumor of the lymphorect System. AFIP 1972;66:13.

5. Mascalchi M, Torselli P, Falaschi F, Dal Pozzo G. MRI of spinal epidural lymphoma. Neuroradiology 1995;37:303-7.

6. McDonald AC, Nicoll JA, Rampling RP. Non-Hodgkin's lymphoma presenting with spinal cord compression: a clinicopathological review of 25 cases. Eur J Cancer 2000;36: 207-13.

7. Wada N, Kohara M, Ikeda J, et al. Diffuse large B-cell lymphoma in the spinal epidural space: a study of the Osaka Lymphoma Study Group. Pathol Res Pract 2010; 206:439-44.
8. Aabo K, Walbom-Jørgensen S. Central nervous system complications by malignant lymphomas: radiation schedule and treatment results. Int J Radiat Oncol Biol Phys 1986;12:197-202.

9. Nelson DF, Martz KL, Bonner H, et al. Non-Hodgkin's lymphoma of the brain: can high dose, large volume radiation therapy improve survival? Report on a prospective trial by the Radiation Therapy Oncology Group (RTOG): RTOG 8315. Int J Radiat Oncol Biol Phys 1992;23:9-17.

10. Perry JR, Deodhare SS, Bilbao JM, Murray D, Muller P. The significance of spinal cord compression as the initial manifestation of lymphoma. Neurosurgery 1993;32:15762. 